\title{
Inhalt, Vol. 14, No. 4, 1991
}

\section{Contents}

Übersichtsarbeiten

Proliferationskinetik und Prognose eines Nierenzellkarzinoms* de Riese, W., Allhoff, E., Werner, M., Stief, C.G.

Atzpodien, J., Kirchner, H

Zur Bewertung von Therapiestudien beim Mammakarzinom

Sauerbrei, W., Schmoor, C, Schumacher, M.

297

Review Articles

Proliferation Kinetics and Prognosis of Renal Cell Carcinoma* de Riese, W., Allhoff, E., Werner, M., Stief, C.G., Atzpodien, J., Kirchner, $\mathrm{H}$ On the Assessment of Quality in Breast Cancer Clinical Trials Sauerbrei, W., Schmoor, C, Schumacher, M

2')7

303

Originalarbeiten

Etoposid, Leukovorin und 5-Flourouracil (ELF) beim fortgeschrittenen Magenkarzinom: Abschließende Ergebnisse einer Phase-II-Studie bei älteren Patienten oder Patienten mit kardialem Risiko*

Stahl, M., Wilke, H., Preusser, P., Fink, U., Achterrath, W., Schöber, C, Köhne-Wömpner, C.H., Harstrick, A., Meyer, H.J., Meyer, J., Lenaz, L., Schmoll, HJ 314

Einfluß von Rekombinantem Granulozyten-Makro-phagen Kolonien-stimulierender Faktor (GMCSF) auf Leukopenie bei AIDS: Eine Untersuchung bei sieben Patienten*

Ottmann, O.G., Goebel, F.-D., Ganser, A., Bogner, J.R., Seipelt, G., Chatterjee, M., Helm, E.B., Hoel-zer, D

319

Original Paper

Etoposide, Leukovorin and 5-Flourouracil (ELF) in Advanced Gastric Carcinoma - Final Results of a Phase-II Study in Elderly Patients or Patients with Cardiac Risk*

Stahl, M., Wilke, H., Preusser, P., Fink, U., Achterrath, W., Schöber, C, Köhne-Wömpner, C.H., Harstrick, A., Meyer, H.J., Meyer, J., Lenaz, L., Schmoll, H.J 
Effect of Recombinant Granulocyte-Macrophage Colony-Stimulating Factor (GM-CSF) on

Leukopenia in AIDS: A Study of Seven Cases*

Ottmann. O.G., Goebel, F.-D., Ganser, A., Bogner, J.R.,

Seipelt, G., Chatterjee, M., Helm, E.B., Hoelzer, D 319

Chirurgische Therapie bei großen Knochenmetastasen im Becken

Spring, W.,Dittmer,H 322

Prospektive multizentrische Phase-IП-Studie mit Doxifluridin (5'dFUR) versus 5-Flourouracil bei Patienten mit fortgeschrittenem kolorektalen Karzinom*

Schuster, D., Heim, M.E., Dombernowski, P., Wood, C,

Queißer, W 333

Surgical Therapie in Extensive Pelvic Bone Metastases

Spring, W.,Dittmer,H

Prospective Multicenter Phase-III Trial of Doxifluridin (5'dFUR) versus 5-Flourouracil in

Patients with Advanced Colorectal Carcinoma*

Schuster, D., Heim, M.E., Dombernowski, P, Wood, C,

Queißer, W

322

333

Kurzmitteilungen

Ansprechraten und Toxizität von Mitomycin-C, Mitoxantron und Methotrexat (3M) bei metastiertem Mammakarzinom*

Porzsolt,F.,Meuret, G 338

Schmerztherapie: Vergleich zweier Bestrahlungs-schemata bei schmerzhaften

Knochenmetastasen*

Karstens, J.H.,Blach, M, Ammon, J 341

Immunzytologische Differenzierung zwischen Leberzellkarzinom und metastasierendem

extragonadalem Keimzelltumor*

Hastka, J.,Bohrer, M.H., Hartung, G., Verbeke, C.S 344

Short Communications

Response Rates and Toxicity of Mitomycin-C, Mitoxantrone, and Methotrexate (3M) in

Advanced Breast Cancer*

Porzsolt,F.,Meuret, G 338

Pain Management Policy: Comparison of Two Irradiation Schedules in Metastatic Bone Pain*

Karstens, J.H., Blach, M., Ammon, J 341

Immunocytological Differentiation of Hepatocellular Carcinoma versus Estragonadal Germ Cell

Tumor*

Hastka. J., Bohrer, M.H., Hartung, G., Verbeke, C.S344

Fortsetzung auf Seite 296

Continued on page 296

Internationale Zeitschrift für Krebsforschung und -behandlung

Band 14, Heft 4, August 1991

Inhalt 


\section{Contents}

\section{Kasuistiken}

Nachweis von Mycobacterium tuberculosis bei atypischer Röntgenmorphologie eines kleinzelligen Bronchialkarzinoms unter Zytostatika-Therapie

Heckmayr, M., Gatzemeier, U 346

Informationen für die Klinik

Kostenvergleich zwischen stationärer und ambulanter Dauerinfusionstherapie

Löffler,T.M.,Weber, F.W 352

Mitteilungen onkologischer Gesellschaften

AIO-Mitteilungen 359

Impressum 293

Inhal-tsverzeichnis 294

Buchbesprechungen $\quad 366$

Industrieforum $\quad 369$

Hinweise für Autoren 377

* diese Arbeit erscheint in englischer Sprache

Casuistic Contributions

Detection of Mycobacterium Tuberculosis with Atypical X-Ray Morphology of a Small-Cell

Bronchial Carcinoma under Cytostatic Therapy

Heckmayr, M., Gatzemeier, U 346

Clinical Information

Cost Comparison Between Inpatient Treatment and Ambulatory Continuous Infusion Therapy

Löffler,T.M., Weber, F.W 352

Reports of Oncological Societies

AIO Reports 359

Imprint 293

Contents - $\quad 294$

Book Reviews 366

Industrial Forum $\quad 369$

Instructions to Authors 377

*this article is printed in English

Beilagenhinweis: Einer Teilauflage dieser Ausgabe liegt das Supplement 1/1991

«Psychoneuroimmunologie und Krebs» bei.

Bibliographîscher Hinweis: Inhaltsverzeichnisse dieser Zeitschrift erscheinen regelmäßig in current contents ${ }^{\circledR}$ sowie in anderen bibliographischen Diensten. 